\title{
General approach to the sensitivity of the optics of an eye to change in elementary parameters with application to the Gaussian optics of a reduced eye
}

\author{
WF Harris*
}

Department of Optometry, University of Johannesburg, P O Box 524, Auckland Park, 2006 South Africa

<wharris@uj.ac.za>

Received 17 April 2009; revised version accepted 27 October 2009

\begin{abstract}
Optical properties of the eye, including the refractive compensation, for example, may change if there are changes in any of the components that make up the eye. The sensitivity to such changes is quantified via the derivative. This paper employs the reduced eye and Gaussian optics to illustrate a general method for the analysis of sensitivity in eyes. The method requires a clear statement of the dependent variable as a function of independent variables. A symbolism is offered that makes the function unambiguous. Sensitivities are determined for the fundamental optical properties, the transference and the corneal-plane refractive
\end{abstract}

compensation of a reduced eye to change in corneal power, curvature and radius of curvature and to change in axial length and index of refraction. Emsley's reduced eye is examined in particular. Its corneal-plane refractive compensation has a sensitivity of $-135 \mathrm{D}$ to change in refractive index, $2.7 \mathrm{D} / \mathrm{mm}$ to change in length and $-1 / 3$ to change in corneal curvature when the other two independent variables are held fixed. The method has the potential to develop guidelines that are useful clinically.

Key words: sensitivity, error, transference, derivative, Gaussian optics, reduced eye

\section{Introduction}

It is sometimes desirable to have insight into the sensitivity of the optical character of the eye to change, error or uncertainty in some component within the eye. How sensitive is refractive compensation, for example, to the length of an eye or to the position or power of an intraocular lens? The purpose of this paper is to provide the basis for a general treatment of sensitivity of the optical properties of eyes, and, indeed, all optical systems, to change or error in some component of the system. The paper lays out the principles in the context of the very simplest of possible eyes, the reduced eye, using the very simplest of optical models, Gaussian optics. By this means one hopes that the principles will come though with greatest clarity.

The mathematical vehicle for the study of variation is calculus. In particular sensitivity to change or errors can be represented by means of the derivative. ${ }^{1-3}$ The objective here is to follow the lead provided by the mathematics in search of a general and systematic routine for the analysis of sensitivity in terms of the derivative. The reduced eye and Gaus- 
sian optics allow illustration of the general ideas in a context simple enough that the results one obtains are obvious in most cases. More complicated models can be handled in the same way but with them the formulae and manipulations risk obscuring the underlying methodology.

An example of a more complicated eye is treated in an accompanying paper ${ }^{4}$ : an otherwise reduced eye containing a thin intraocular lens is examined and the sensitivities of its refractive compensation to change in power and axial position are calculated.

It is the author's view that the method described here has the potential to produce guidelines that are useful to the clinician. However it is not the purpose of this paper to seek such guidelines.

We begin by defining sensitivity as a derivative. The usual symbolism used in mathematics is not designed to enable one easily to keep track of dependent and independent variables. Accordingly a symbolism is proposed for functions and derivatives that displays these variables explicitly and provides a constant reminder to the user. Formally there is nothing new in the mathematics; all we are doing is using the usual rules of differentiation. It is easy in multi-variable analysis to get bogged down in mathematical technicalities. We gloss over them in order to get the job done. Having reviewed the necessary Gaussian optics the paper examines the reduced eye in particular. Functions representing its fundamental properties, its transference and its corneal-plane refractive compensation are defined with the elementary optical properties as independent variables. They in turn define a natural set of sensitivities of these properties to change in one of the independent variables. Functions and sensitivities are also defined in terms of what we call geometrical and indicial variables in the system. Finally the treatment is made more concrete with application to Emsley's reduced eye in particular.

\section{Sensitivity as derivative}

Suppose some scalar optical property $z$ of an eye can be represented as a function $f$ of the three independent scalar variables $w, x$ and $y$. One writes

$$
z=f(w, x, y)
$$

(For example, $z$ might be the eye's refractive compensation, $w$ the curvature of the cornea, $x$ the power of the natural lens and $y$ the length of the eye.) The mathematics, then, immediately defines three (first) derivatives, one for each independent variable. The derivative $\frac{\partial f}{\partial w}$ is the sensitivity of $z$ to change in $w$ while $x$ and $y$ are held fixed. Similarly $\frac{\partial f}{\partial x}$ is the sensitivity of $z$ to change in $x$ with $w$ and $y$ held fixed and $\frac{\partial f}{\partial y}$ the sensitivity of $z$ to change in $y$ with $w$ and $x$ constant. (Derivatives, like these, of functions of more than one variable are often called partial derivatives.)

For a sufficiently small increment $\Delta w$ in $w$ there is an increment $\Delta z$ in $z$ given by

$\Delta z \approx \frac{\partial f}{\partial w} \Delta w$.

The symbol $\approx$ should be read 'approximately equal'. Replacing $w$ by $x$ in Equation 2 gives the increment in $z$ in terms of an increment in $x$ and similarly for $y$.

Symbols like $f$ for the function are arbitrary, and like the symbols for the derivatives, do not lend themselves to systematic treatments of variation of the many different optical properties of eyes and their dependence on elements within the system. Instead new symbols are constructed in the next section that facilitate such analyses.

\section{Symbolism}

An eye has many optical properties each of which depends on many parameters. In order to handle these many quantities we shall need a systematic symbolism which displays the dependent and independent variables explicitly. We shall make use of the symbolism devised elsewhere. ${ }^{5-7}$ Instead of $f$ we shall construct a symbol using the independent variables attached as superscripts to the dependent variable, that is, we shall use $z^{w x y}$ instead of $f$ for the function above.

If the independent variables are assigned particular values then the value of the function can be specified with the values of the independent variables in parentheses following the symbol for the function. For 
example, for $w=w_{0}, x=x_{0}$ and $y=y_{0}$ the value of the function $z^{w x y}$ is $z^{w x y}\left(w_{0}, x_{0}, y_{0}\right)$. Thus one can write

$$
z=z^{w x y}\left(w_{0}, x_{0}, y_{0}\right)
$$

The order of the superscripted variables is arbitrary. However the order of the values in parentheses must match that of the superscripted variables. When the values of the independent variables are unspecified we will often write

$$
z=z^{w x y}
$$

in which we shall regard $(w, x, y)$ as understood after the symbol $z^{w x y}$.

Derivatives of a function of one variable are often indicated by means of a prime attached to the symbol for the function as in $f^{\prime}$. We shall do the same for functions of several variables but we shall attach the prime to the appropriate superscripted variable. For

example, for the derivative $\frac{\partial f}{\partial w}$ we shall instead write $z^{w^{\prime} x y}$

The symbol $z^{w x y}$ immediately implies three derivatives, namely $z^{w^{\prime} x y}, z^{w x^{\prime} y}$ and $z^{w x y^{\prime}}$. They are the sensitivities of $z$ to change in each of $w, x$ and $y$. And they are themselves functions of $w, x$ and $y$. At particular values of the independent variables the sensitivity has a value given, for example, by $z^{w^{\prime} x y}\left(w_{0}, x_{0}, y_{0}\right)$.

All the independent variables, and their values, provide, as it were, a context for the sensitivity, and the context must be clear at all times. Because it is quite possible for $z^{w^{\prime} x y}$ to be different from $z^{w^{\prime} x u}$, where $u$ is another independent variable, and even $z^{w^{\prime} x}$, one cannot in general talk simply of the sensitivity of $z$ to change in $x$ with nothing said, or at least implied, about the other independent variables. Constancy of the other independent variables is the mathematical expression of the notion of ceteris paribus ("with other things equal') in the economic literature ${ }^{8}$. This is an important issue when it comes to optical applications. The mathematical notation we use will be a firm reminder of precisely what is held constant as much as of what is changing.
With the notation described here Equation 2 becomes

$\Delta z \approx z^{w^{\prime} x y} \Delta w$

or, if the values of the independent variables are specified,

$\Delta z \approx z^{w^{\prime} x y}\left(w_{0}, x_{0}, y_{0}\right) \Delta w$

for example.

Curve $\mathrm{C}$ in Figure 1 provides a graphical illustration. It represents the function $z^{w x y}$ for some fixed $x$ and $y . w$ and $z$ are plotted along the $\mathrm{W}$, horizontal, and $\mathrm{Z}$, vertical, axes. The $\mathrm{X}$ and $\mathrm{Y}$ axes are perpendicular to the plane of the paper and each other. We are thinking of a four-dimensional space. Figure 1 is a two-dimensional cross-section, at particular values of $x$ and $y$, of the four-dimensional space. $\mathrm{T}$ is the tangent to curve $\mathrm{C}$ at point $\mathrm{P}$, that is, at the particular value of $w$. Its slope is the derivative $z^{w^{\prime} x y}$, the sensitivity of $z$ to change in $w$ at constant $x$ and $y$. The sensitivity is positive at $\mathrm{P}$. At $\mathrm{Q}$ the sensitivity is less. At the maximum in Figure 1 the sensitivity is zero and beyond it the sensitivity is negative.

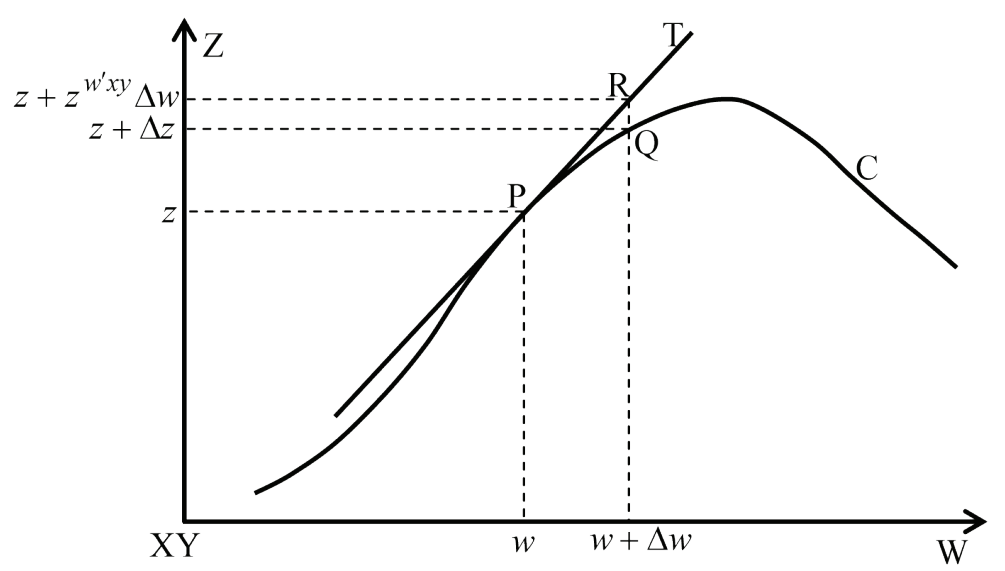

Figure 1 A property $z$ is expressed as a function $z^{w x y}$ of three independent parameters, $w, x$, and $y$. Here the dependence of $\mathrm{z}$ on $w$ for some fixed values of $x$ and $y$ is shown by means of curve C. In particular, an increment $\Delta w$ in $w$ results in an increment $\Delta z$ in $z$. The increment in $w$ takes one from point $\mathrm{P}$ on $\mathrm{C}$ to point Q. T is the tangent to $\mathrm{C}$ at point $\mathrm{P}$. Its slope is the derivative of $z^{w x y}$ with respect to $w$, that is, $z^{w^{\prime} x y}$. It is also the sensitivity of $z$ to change in $w$ with $x$ and $y$ held fixed. $z^{w^{\prime} x y} \Delta w$ is an estimate of $\Delta z$ (Equation 5).

Suppose there is some small positive increment $\Delta w$ in $w$. This in effect shifts one to the right along 
axis $\mathrm{W}$ in Figure 1 by the amount $\Delta w$ to the position $w+\Delta w$ and along curve $\mathrm{C}$ from $\mathrm{P}$ to $\mathrm{Q}$. At this incremented value of $w$ variable $z$ has the value represented by point $\mathrm{Q}$, that is, $z+\Delta z$. Thus an increment $\Delta w$ in $w$, while $x$ and $y$ are held fixed, is associated with an increment $\Delta z$ in $z . \quad z^{w^{\prime} x y} \Delta w$ is an increase in $z$ from the value shown by $\mathrm{P}$ to the value shown by $\mathrm{R}$, and, provided the curve is not too strongly curved, may be a reasonable estimate of $\Delta z$ in accordance with Equation 5. The smaller $\Delta w$ the better the estimate.

Sensitivity is not constant in general; it itself has a sensitivity to change in the independent variables. A sensitivity of a sensitivity is what we shall call a second-order sensitivity. It is a derivative of a derivative, that is, a second derivative. The three sensitivities of the sensitivity $z^{w^{\prime} x y}$, for example, are the second-order sensitivities $z^{w^{\prime \prime} x y}, z^{w^{\prime} x^{\prime} y}$ and $z^{w^{\prime} x y^{\prime}}$. (Implicit in this symbolism is the technical requirement that the function is a $\mathrm{C}^{2}$ function, that is, every second-order derivative exists and is continuous. ${ }^{9}$ This is not the only technicality that we are going to gloss over in this paper. They are issues that can be raised if necessary in the future.) The notation can be extended to derivatives of higher order if necessary.

If a particular sensitivity is 0 (at the maximum of $\mathrm{C}$ in Figure 1 for example) we shall say the dependent variable is insensitive to change in the independent variable in question at the particular values of all the independent variables.

From $z^{w x y}(w, x, y)$ and the derivative $z^{w^{\prime} x y}(w, x, y)$ we can estimate $z$ for an increment $\Delta w$ in $w$ with $x$ and $y$ constant. The estimated value $z_{\approx}$ of $z$ is given by

$z_{\approx}=z+z^{w^{\prime} x y} \Delta w$.

Then the error in the estimate is the estimated value minus the true value, that is,

$$
\delta_{\mathrm{e}} z=z_{\approx}-z^{w x y}(w+\Delta w, x, y) .
$$

We turn now to some necessary results from Gaussian optics.

\section{Gaussian optics}

In Gaussian optics the transference of a centred $C=-K\left(n-n_{0}\right)$ system can be represented as the $2 \times 2$ matrix ${ }^{10-16}$

$$
\mathbf{S}=\left(\begin{array}{ll}
A & B \\
C & D
\end{array}\right)
$$

$\mathbf{S}$ is also called the system matrix ${ }^{12,16}$ and the raytransfer matrix ${ }^{13}$ and its entries the Gaussian constants of the optical system ${ }^{11}$. There are inconsistencies in the literature in the arrangement of the entries within the matrix and in the signs of the off-diagonal entries. We refer to the entries as the four fundamental properties of the system and distinguish $A$ as the dilation, $B$ the disjugacy, $C$ the divergence and $D$ the divarication. ${ }^{17} \mathbf{S}$ is symplectic which implies that its determinant is $1 .^{10-15}$ By definition ${ }^{18}$ the power $F$ of the system is

$$
F:=-C \text {. }
$$

Consider systems $S_{1}$ and $S_{2}$ with transferences $\mathbf{S}_{1}$ and $\mathbf{S}_{2}$ respectively. Suppose they are juxtaposed in order to form the compound system $\mathrm{S}_{1} \mathrm{~S}_{2}$. Then the compound system has transference ${ }^{10-16}$

$$
\mathbf{S}=\mathbf{S}_{2} \mathbf{S}_{1}
$$

In particular a homogeneous gap of reduced width $\zeta$ has transference ${ }^{10-16}$

$$
\mathbf{S}=\left(\begin{array}{ll}
1 & \zeta \\
0 & 1
\end{array}\right)
$$

In terms of the width $z$ of the gap and the index of refraction of the medium $n$

$$
\zeta=z / n
$$

A refracting surface of divergence $C$ has transference $^{10-16}$

$$
\mathbf{S}=\left(\begin{array}{ll}
1 & 0 \\
C & 1
\end{array}\right)
$$

In terms of its curvature $K$ a surface has divergence ${ }^{10-16}$

$$
C=-K\left(n-n_{0}\right)
$$


where $n$ is the index immediately downstream and $n_{0}$ or the index immediately upstream from the surface.

In the case of an eye in particular the dilation $A$ in the transference is a measure of ametropia. The corneal-plane refractive compensation (also called the refraction or the refractive state, et cetera) of the eye is ${ }^{17}$

$$
F_{0}=A / B \text {. }
$$

\section{Reduced eye}

Figure 2 shows a reduced eye. It consists only of a single refracting surface $\mathrm{K}$, the 'cornea', of curvature $K$ and a retina $\mathrm{R}$ separated by a homogeneous gap of width $z$ and index of refraction $n$. $Z$ is a longitudinal axis. $\mathrm{T}_{0}$ and $\mathrm{T}$ are two transverse planes. $\mathrm{T}_{0}$ is the entrance plane of the optical system of the eye; it is immediately in front of the single-surface cornea K. The exit plane $\mathrm{T}$ is immediately in front of the retina $\mathrm{R}$. The medium in front of the eye is air (index 1).

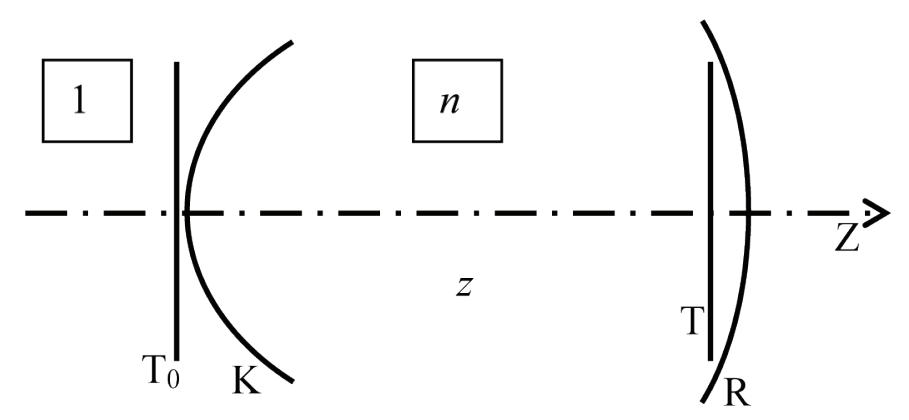

Figure 2 A reduced eye consisting of a refracting surface $\mathrm{K}$ of curvature $K$ and a homogeneous medium of length $z$ and index $n$. The optical system of the eye is from the entrance plane $T_{0}$, immediately in front of the cornea, to the exit plane $\mathrm{T}$, immediately in front of the retina $\mathrm{R} . \mathrm{Z}$ is a longitudinal axis. The medium in front of the eye is air with index 1 .

Equation 13 gives the reduced length of the eye and Equation 15 the divergence of the cornea:

$C=-K(n-1)$.

Let refracting surface $\mathrm{K}$ be system $\mathrm{S}_{1}$ and the gap system $\mathrm{S}_{2}$. Then, from Equations 12, 14 and 11 the eye has transference

$$
\mathbf{S}=\left(\begin{array}{ll}
1 & \zeta \\
0 & 1
\end{array}\right)\left(\begin{array}{ll}
1 & 0 \\
C & 1
\end{array}\right)
$$

$$
\mathbf{S}=\left(\begin{array}{cc}
1+\zeta C & \zeta \\
C & 1
\end{array}\right)
$$

$\zeta$ and $C$ are scalars that characterize the optics of the two elementary systems making up the reduced eye. We shall distinguish them as the elementary optical parameters of the eye.

\section{Sensitivity of a reduced eye to change in its ele- mentary optical parameters}

Comparison of Equations 19 and 9 shows that the ametropia of the eye is given by

$A=1+\zeta C$

Equation 20 expresses $A$ as a function of the two independent variables $\zeta$ and $C$. In keeping with the notation described above we represent the function by means of the symbol $A^{\zeta C}$. There are two sensitivities $A^{\zeta^{\prime} C}$, the sensitivity of $A$ to change in $\zeta$ with $C$ constant, and $A^{\zeta C^{\prime}}$, the sensitivity of $A$ to change in $C$ with $\zeta$ constant.

From Equation 20 we see that the sensitivities are given by

$A^{\zeta^{\prime} C}=C$

and

$A^{\zeta C^{\prime}}=\zeta$.

Typically $C$ is in dioptres and $\zeta$ in metres. $A$ has no units. Thus the sensitivity of $A$ to change in $\zeta$ would typically be in dioptres or reciprocal metres. The sensitivity of $A$ to change in $C$ on the other hand would typically be in metres or reciprocal dioptres.

We note in passing that the sensitivity $A^{\zeta^{\prime} C}$ is not constant; it itself has two sensitivities, the second-order sensitivities $A^{\zeta^{\prime \prime} C}=0$ and $A^{\zeta^{\prime} C^{\prime}}=1$ which are constant.

Consider now a small increment $\Delta \zeta$ in $\zeta$. Following Equation 5 we see that the increment causes an increment $\triangle A$ in $A$ given by

$\Delta A \approx A^{\zeta^{\prime} C} \Delta \zeta$. 
From Equation 21

$$
\Delta A \approx C \Delta \zeta \text {. }
$$

(Actually Equation 20 shows that the equality is exact and that $\approx$ could be replaced by $=$ in this case.) Similarly, a small increment $\Delta C$ in divergence $C$ causes an increment

$\Delta A \approx A^{\zeta C^{\prime}} \Delta C$

or, because of Equation 22,

$$
\Delta A \approx \zeta \Delta C \text {. }
$$

As has been done above for the ametropia $A$ the same can be done for the remaining three fundamental properties. Again from Equations 19 and 9 for disjugacy $B$

$B=\zeta$.

It follows that its two sensitivities are

$$
\begin{aligned}
& B^{\zeta^{\prime} C}=1 \\
& \text { and } \\
& B^{\zeta C^{\prime}}=0 .
\end{aligned}
$$

This implies the obvious fact that the eye's disjugacy $B$ has unit sensitivity to change in reduced length $\zeta$ and is insensitive to change in the divergence $C$ of the cornea. Similarly, one obtains the two sensitivities of divergence

$$
C^{\zeta^{\prime} C}=0
$$

and

$$
C^{\zeta C^{\prime}}=1
$$

showing that the eye's divergence is insensitive to change in reduced length but has unit sensitivity to change in the divergence of the cornea. Finally Equation 19 shows that

$$
D^{\zeta^{\prime} C}=0
$$

and

$$
D^{\zeta C^{\prime}}=0 \text {, }
$$

or, in other words, the eye's divarication $D$ is insensitive to change in reduced length of the eye and to change in the divergence of the cornea.

The sensitivity of derived properties can be treated similarly. Consider the refractive compensation. From Equations 16 and 19 the corneal-plane refractive compensation of the reduced eye is

$F_{0}=1 / \zeta+C$

The function is $F_{0}^{\zeta C}$. Hence there are the two sensitivities,

$F_{0}^{\zeta^{\prime} C}=-1 / \zeta^{2}$

and

$F_{0}^{\zeta C^{\prime}}=1$

the latter being precisely what one would expect.

All the separate results for the fundamental properties can be combined in one if we view Equation 19 as a matrix function $\mathbf{S}^{\zeta C}$ of the two independent scalar variables $\zeta$ and $C$. Such functions are differentiated simply by differentiating the separate scalar functions within the matrix. ${ }^{2,3,19}$ (The matrix derivatives used here are of the type Magnus and Neudecker3 represent as $\frac{\partial \mathbf{S}(\zeta)}{\partial \zeta}$ as opposed to those they represent as $\operatorname{DS}(\zeta)$.) Differentiating with respect to $\zeta$ gives

$$
\mathbf{S}^{\zeta^{\prime} C}=\left(\begin{array}{ll}
C & 1 \\
0 & 0
\end{array}\right),
$$

the sensitivity of the eye's transference to change in the eye's reduced length for constant $C$, and differentiating with respect to $C$ gives

$$
\mathbf{S}^{\zeta C^{\prime}}=\left(\begin{array}{ll}
\zeta & 0 \\
1 & 0
\end{array}\right),
$$

the sensitivity of the eye's transference to change in divergence of the cornea for constant reduced length. Equation 37 contains Equations 21, 28, 30 and 32 while Equation 38 contains Equations 22, 29, 31 and 33. Note that, while the transference is symplectic, and so has unit determinant, its sensitivities are singular. Differentiation, one might say, destroys symplecticity. 
Instead of obtaining the sensitivities of individual properties by differentiation, as done above for $F_{0}$, one can obtain them from the transference and its sensitivity without further differentiation. For example, from Equation 16, one can write

$$
F_{0}^{\zeta C}=A^{\zeta C} / B^{\zeta C} \text {. }
$$

Then, from the well-known rule for differentiation of a quotient, the sensitivity of the corneal-plane refractive compensation to change in reduced length of the eye while the divergence of the cornea is held fixed is

$$
F_{0}^{\zeta^{\prime} C}=\frac{B^{\zeta C} A^{\zeta^{\prime} C}-A^{\zeta C} B^{\zeta^{\prime} C}}{\left(B^{\zeta C}\right)^{2}} .
$$

$A^{\zeta C}$ and $B^{\zeta C}$ are the top-left and top-right entries, respectively, of $\mathbf{S}$ in Equation 19 (also see Equations 20 and 27). $A^{\zeta^{\prime} C}$ and $B^{\zeta^{\prime} C}$ are the corresponding entries of the sensitivity $\mathbf{S}^{\zeta^{\prime} C}$ of Equation 37 (also see Equations 21 and 28). Substitution of these values into Equation 40 leads to the equation obtained before (Equation 35).

So far the independent variables have been the elementary optical parameters of the system $\zeta$ and $C$. There are other possibilities. From Equations 19 and 10

$$
\mathbf{S}=\left(\begin{array}{cc}
1-\zeta F & \zeta \\
-F & 1
\end{array}\right)
$$

which now defines the function $\mathbf{S}^{\zeta F}$, the transference $\mathrm{S}$ as a function of reduced length $\zeta$ and power $F$. So we have the sensitivity of the eye's transference to change in reduced length,

$$
\mathbf{S}^{\zeta^{\prime} F}=\left(\begin{array}{cc}
-F & 1 \\
0 & 0
\end{array}\right),
$$

and to change in the power of the cornea,

$$
\mathbf{S}^{\zeta F^{\prime}}=\left(\begin{array}{cc}
-\zeta & 0 \\
-1 & 0
\end{array}\right)
$$

with the other independent variable held constant. One notes that
$\mathbf{S}^{\zeta^{\prime} F}=\mathbf{S}^{\zeta^{\prime} C}$

whereas

$\mathbf{S}^{\zeta F^{\prime}}=-\mathbf{S}^{\zeta C^{\prime}}$

which is not unexpected. Also

$F_{0}^{\zeta^{\prime} F}=F_{0}^{\zeta^{\prime} C}$

and

$F_{0}^{\zeta F^{\prime}}=-F_{0}^{\zeta C^{\prime}}$

Sensitivities of the eye to changes in the elementary optical parameters assume, as we have seen, a particularly simple form. However they may not be the most useful in practice. Another set of sensitivities is described next.

\section{Sensitivity of a reduced eye to geometrical and in- dicial changes}

Let us now examine the sensitivity of the optical character of the reduced eye of Figure 2 to change in the curvature $K$ of its cornea, its length $z$ and its index of refraction $n$. Collectively we shall refer to them as the geometrical and indicial parameters of the eye. Substituting from Equations 13 and 15 into Equation 19 we obtain the transference

$\mathbf{S}=\left(\begin{array}{cc}1-z K+z K / n & z / n \\ -K(n-1) & 1\end{array}\right)$

expressed now as a function $\mathbf{S}^{n z K}$ of three independent variables $n, z$ and $K$. It is not as neat as the transference expressed in terms of the elementary optical parameters (Equation 19).

With its three independent variables $\mathbf{S}^{n z K}$ defines three sensitivities for the transference of the reduced eye:

$\mathbf{S}^{n^{\prime} z K}=\left(\begin{array}{cc}-z K / n^{2} & -z / n^{2} \\ -K & 0\end{array}\right)$,

the sensitivity to change in index of refraction,

$\mathbf{S}^{n z^{\prime} K}=\left(\begin{array}{cc}-K(n-1) / n & 1 / n \\ 0 & 0\end{array}\right)$ 
the sensitivity to change in length of the eye, and

$$
\mathbf{S}^{n z K^{\prime}}=\left(\begin{array}{cc}
-z(n-1) / n & 0 \\
-(n-1) & 0
\end{array}\right),
$$

the sensitivity to change in corneal curvature.

From Equation 34 the corneal-plane refractive compensation expressed in terms of the geometrical and indicial parameters, is

$$
F_{0}=n / z-(n-1) K \text {. }
$$

So we have three sensitivities of the compensation,

$F_{0}^{n^{\prime} z K}=1 / z-K$,

to change in index,

$F_{0}^{n z^{\prime} K}=-n / z^{2}$,

to change in length of the eye, and

$F_{0}^{n z K^{\prime}}=-(n-1)$

to change in curvature of the cornea. In each case the notation reminds us which other independent variables are fixed.

In terms of the radius of curvature $r$ of the cornea instead of curvature $K$ Equation 48 becomes

$\mathbf{S}=\left(\begin{array}{cc}1-z / r+z /(n r) & z / n \\ -(n-1) / r & 1\end{array}\right)$

which leads to the three sensitivities

$\mathbf{S}^{n^{\prime} z r}=\left(\begin{array}{cc}-z /\left(n^{2} r\right) & -z / n^{2} \\ -1 / r & 0\end{array}\right)$,

$\mathbf{S}^{n z^{\prime} r}=\left(\begin{array}{cc}-(n-1) /(n) & r^{1 / n} \\ 0 & 0\end{array}\right)$

and

$\mathbf{S}^{n z r^{\prime}}=\left(\begin{array}{cc}z(n-1) /\left(n r^{2}\right) & 0 \\ (n-1) / r^{2} & 0\end{array}\right)$.

From Equation 52

$F_{0}=n / z-(n-1) / r$

and so

$$
\begin{aligned}
& F_{0}^{n^{\prime} z r}=1 / z-1 / r, \\
& F_{0}^{n z^{\prime} r}=-n / z^{2}
\end{aligned}
$$

and

$F_{0}^{n z r^{\prime}}=(n-1) / r^{2}$.

As expected $F_{0}^{n^{\prime} z r}=F_{0}^{n^{\prime} z K}$ and $F_{0}^{n z^{\prime} r}=F_{0}^{n z^{\prime} K}$. On the other hand sensitivity $F_{0}^{n z r^{\prime}}$ depends on the curvature of the cornea while sensitivity $F_{0}^{n z K^{\prime}}$ does not.

In the next section we use Emsley's reduced eye to put numbers to these equations.

\section{Emsley's reduced eye}

Atchison and Smith list parameters for Emsley's reduced eye: ${ }^{2}$ its length is $z=22.22222 \mathrm{~mm}$, the index is $n=4 / 3$ and its cornea has power $F=60 \mathrm{D}$.

From this data one obtains the reduced length (Equation 13) of the eye: $\zeta=16.6667 \mathrm{~mm}$. From Equation 41 we obtain the transference of the eye,

$$
\mathbf{S}=\left(\begin{array}{cc}
10^{-7} & 16.6667 \mathrm{~mm} \\
-60 \mathrm{D} & 1
\end{array}\right)
$$

From Equations 42 and 43 we obtain the two sensitivities

$\mathbf{S}^{\zeta^{\prime} F}=\left(\begin{array}{cc}-60 \mathrm{D} & 1 \\ 0 & 0\end{array}\right)$

and

$\mathbf{S}^{\zeta F^{\prime}}=\left(\begin{array}{cc}-16.6667 \mathrm{~mm} & 0 \\ -1 & 0\end{array}\right)$,

the sensitivities to changes in the elementary parameters $\zeta$ and $F$ respectively with the other one held fixed.

From Equation 34 one sees that the corneal-plane refractive compensation of the eye is $F_{0}=6 \times 10^{-6} \mathrm{D}$. (The eye has ametropia $10^{-7}$. Clinically it would be regarded as emmetropic.) The sensitivity of the corneal-plane refractive compensation to change in re- 
duced length with constant corneal power is

$F_{0}^{\zeta^{\prime} F}=-3600 \mathrm{D}^{2}$

from Equations 46 and 35, a result that could also be obtained from $\mathbf{S}$ (Equation 64) and its sensitivity $\mathbf{S}^{\zeta^{\prime} F}$ (Equation 65) using Equation 40 with $C$ replaced by $F$ throughout. The result could also be expressed as $-3600 \mathrm{D} / \mathrm{m}$ or $-3.60 \mathrm{D} / \mathrm{mm}$. In other words an increase in reduced length of a millimeter causes a decrease in refractive compensation of about 3.6 D.

From Equations 47 and 36 the sensitivity of the compensation to change in corneal power is

$F_{0}^{\zeta F^{\prime}}=-1$.

That might be thought of as $-1 \mathrm{D} / \mathrm{D}$. An increase in corneal power of $1 \mathrm{D}$ causes a decrease in cornealplane refractive compensation by $1 \mathrm{D}$, a conclusion that is obviously correct.

Consider now sensitivities to changes in the geometrical and indicial parameters of the eye. From Equation 15 we find that the curvature of the cornea is $K=180 \mathrm{D}$. Then from Equations 49,50 and 51 we obtain the sensitivities of the transference to change in each of the three independent variables

$$
\begin{aligned}
& \mathbf{S}^{n^{\prime} z K}=\left(\begin{array}{cc}
-2.25 & -12.5 \mathrm{~mm} \\
-180 \mathrm{D} & 0
\end{array}\right), \\
& \mathbf{S}^{n z^{\prime} K}=\left(\begin{array}{cc}
-45 \mathrm{D} & 0.75 \\
0 & 0
\end{array}\right)
\end{aligned}
$$

and

$\mathbf{S}^{n z K^{\prime}}=\left(\begin{array}{cc}-5.6 \mathrm{~mm} & 0 \\ -1 / 3 & 0\end{array}\right)$

with the other two held fixed. From Equation 53 (or from Equations 64 and 69) one finds that $F_{0}^{n^{\prime} z K}=-135 \mathrm{D}$. Thus an increase in index by 0.001 causes a decrease in corneal-plane refractive compensation by about $0.135 \mathrm{D}$. From Equation 54 $F_{0}^{n z^{\prime} K}=-2700 \mathrm{D}^{2}$ or $-2700 \mathrm{D} / \mathrm{m}$ or $-2.7 \mathrm{D} / \mathrm{mm}$. Thus an increase in length of the eye of $1 \mathrm{~mm}$ causes a decrease in refractive compensation of about $2.7 \mathrm{D}$. From Equation $55 F_{0}^{n z K^{\prime}}=-1 / 3$. Hence an increase in curvature of $1 \mathrm{D}$ causes a decrease in compensation by about $1 / 3 \mathrm{D}$.

From Equation 7 one can use the sensitivity to estimate the value of a property associated with a change in the independent variable. Equation 8 gives the error of the estimate. For example, for an increase in reduced length by $\Delta \zeta=1 \mathrm{~mm}$ and the sensitivity $\mathbf{S}^{\zeta^{\prime} F}$ of Equation 65 we estimate a new transference of

$\mathbf{S}_{\approx}=\left(\begin{array}{cc}10^{-7} & 16.6667 \mathrm{~mm} \\ -60 \mathrm{D} & 1\end{array}\right)+(0.001 \mathrm{~m})\left(\begin{array}{cc}-60 \mathrm{D} & 1 \\ 0 & 0\end{array}\right)$,

that is

$\mathbf{S}_{\approx}=\left(\begin{array}{cc}-0.0600 & 17.6667 \mathrm{~mm} \\ -60 \mathrm{D} & 1\end{array}\right)$

Equation 41, with $\zeta$ increased by $1 \mathrm{~mm}$, gives the true transference. In this case it is exactly the same as the estimated value. Hence the error in the estimate is a null matrix.

Using Equation 69 for the sensitivity $\mathbf{S}^{n^{\prime} z K}$ we find that an increase in index $n$ by 0.001 gives an estimated transference of

$\mathbf{S}_{\approx}=\left(\begin{array}{cc}-0.00225 & 16.6542 \mathrm{~mm} \\ -60.18 \mathrm{D} & 1\end{array}\right)$,

the error being

$\delta_{\mathrm{e}} \mathbf{S}=\left(\begin{array}{cc}-1.686 \times 10^{-6} & -9 \times 10^{-9} \mathrm{~m} \\ 0 & 0\end{array}\right)$.

The estimated transference $\mathbf{S}_{\approx}$ has determinant $1-2.25 \times 10^{-6}$ which illustrates the fact that transferences estimated this way are not necessarily symplectic.

\section{Concluding remarks}

Mathematics is a hard taskmaster. And jolly good too! It does not sanction loose thought. It does not allow one simply to say that the sensitivity of refractive compensation to change in length of the eye is so much. It requires one also to be just as specific about what is not changing, for it is quite possible that the sensitivity of the refractive compensation to a change in length differs according to what is kept 
constant although this is not apparent from the simple eye examined here. We have adopted a notation for a function and its derivatives that makes the independent variables explicit and a constant reminder to the user. The derivatives are the sensitivities. We note in passing that second derivatives are sensitivities of sensitivities.

As an example above shows an estimated transference may depart from symplecticity. This suggests that sensitivities of transferences should be treated with caution when taken for the system as a whole. They are useful nevertheless in that their entries can be used for calculating sensitivities of particular properties as is illustrated by Equation 40 for the sensitivity of the refractive compensation to change in reduced length.

We have used Gaussian optics to examine the question of sensitivity in the case of the reduced eye. In particular we have looked at the sensitivities of the fundamental optical properties of the eye and also the corneal-plane refractive compensation. The degree to which natural eyes approach a reduced eye will be the degree to which the equations obtained here apply to actual eyes. One imagines, though, that the results obtained for Emsley's reduced eye in particular (for example, the corneal-plane refractive compensation has a sensitivity of $-135 \mathrm{D}$ to change in refractive index, $-2.7 \mathrm{D} / \mathrm{mm}$ to change in length and $-1 / 3$ to change in corneal curvature when the other two independent variables are held fixed) do hold, roughly at least, for natural eyes. However it is not these results that are the central point of this paper; the point is the method. The method is general, systematic and complete. Although the algebraic manipulations become increasingly messy as the eye grows in complexity the method can, in principle, be applied to any eye of known structure and it is not limited to Gaussian optics. It is systematic in that it requires one to formulate clear functions with unambiguous statements of what are the independent variables and which of them are changing. It is complete in two senses. It is complete in that, once the function is defined, all of the (first-order) sensitivities are automatically defined as well. And it is also complete in the sense that it can be applied to any optical property of the system, eye or not eye.
An accompanying paper ${ }^{4}$ treats the sensitivity of a simplified eye to the change in power and axial displacement of a thin intraocular lens implanted in it.

\section{Acknowledgements}

I thank $\mathrm{RD}$ van Gool for continuing discussions and the National Research Foundation for support.

\section{References}

1. Taylor JR. An Introduction to Error Analysis, 2nd ed. University Science Books, Sausolita, CA, 1997, pp 64 et seq.

2. Golub GH, van Loan CF. Matrix Computations, 3rd ed. Johns Hopkins Univ Press, Baltimore, MD, 1996, pp 51, 53.

3. Magnus JR, Neudecker H. Matrix Differential Calculus with Applications in Statistics and Econometrics, revised ed. Wiley, Chichester, 1999, pp 171, 306 et seq.

4. Harris WF, van Gool RD. Sensitivity of the corneal-plane refractive compensation to change in power and axial position of an intraocular lens. S Afr Optom 200968 175-178

5. Harris WF. A proposed mathematical notation for use in teaching thermodynamics. ChemSA 19817 259-260.

6. Harris WF. Equilibrium thermodynamics - revisited. Chem Eng Ed 19927 29, 37.

7. Harris WF. Invariance of ophthalmic properties under spherocylindrical transposition. Optom Vis Sci 199774 459-462.

8. Wetzetstein ME. Microeconomic Theory: Concepts and Connections. South-Western, Mason, OH, 2005, p 83.

9. Bleeker D, Csordas G. Basic Partial Differential Equations. International Press, Cambridge, MA, 1996, p 23.

10. Guillemin V, Sternberg S. Symplectic Techniques in Physics. Cambridge University Press, Cambridge, 1984, p 7 et seq.

11. Brouwer W. Matrix Methods in Optical Instrument Design. Benjamin, New York, 1964, pp 15 et seq.

12. Blaker, JW. Geometric Optics: the Matrix Theory. Dekker, new York, 1971, p 29 et seq.

13. Gerrard A, Burch JM. Introduction to Matrix Methods in Optics. Wiley, London, 1975, p 39.

14. Ditteon R. Modern Geometrical Optics. Wiley, New York, 1998, p 176 et seq.

15. Kloos G. Matrix Methods for Optical Layout. SPIE Press, Bellingham, WA, 2007, p 3 et seq.

16. Sharma KK. Optics: Principles and Applications. Elsevier, Amsterdam, 2006, p 141 et seq.

17. Harris WF. A unified paraxial approach to astigmatic optics. Optom Vis Sci 199976 480-499.

18. Harris WF. Dioptric power: its nature and its representation in three- and four-dimensional space. Optom Vis Sci 1997 74 349-366.

19. Lütkepohl H. Handbook of Matrices. Wiley, Chichester, 1996, p 237.

20. Atchison DA, Smith G. Optics of the Human Eye. Butterworth-Heinemann, Oxford, 2000, p 252. 